\title{
Microstructural characteristics and mechanical properties of magnetron sputtered nanocrystalline TiN films on glass substrate
}

\author{
VIPIN CHAWLA ${ }^{\dagger, \dagger \dagger}$, R JAYAGANTHAN ${ }^{\dagger, *}$ and RAMESH CHANDRA ${ }^{\dagger \dagger}$ \\ ${ }^{\dagger}$ Department of Metallurgical and Materials Engineering and Centre of Nanotechnology, \\ ${ }^{\dagger}$ Nano Science Laboratory, Institute Instrumentation Centre, Indian Institute of Technology Roorkee, \\ Roorkee 247 667, India
}

MS received 20 June 2008; revised 26 July 2008

\begin{abstract}
Nanocrystalline TiN thin films were deposited on glass substrate by d.c. magnetron sputtering. The microstructural characteristics of the thin films were characterized by XRD, FE-SEM and AFM. XRD analysis of the thin films, with increasing thickness, showed the (200) preferred orientation up to $1.26 \mu \mathrm{m}$ thickness and then it transformed into (220) and (200) peaks with further increase in thickness up to $2.83 \mu \mathrm{m}$. The variation in preferred orientation was due to the competition between surface energy and strain energy during film growth. The deposited films were found to be very dense nanocrystalline film with less porosity as evident from their FE-SEM and AFM images. The surface roughness of the TiN films has increased slightly with the film thickness as observed from its AFM images. The mechanical properties of TiN films such as hardness and modulus of elasticity $(E)$ were investigated by nanoindentation technique. The hardness of TiN thin film was found to be thickness dependent. The highest hardness value (24 GPa) was observed for the TiN thin films with less positive micro strain.
\end{abstract}

Keywords. TiN thin films; nanoindentation; microstructural characterization.

\section{Introduction}

Nanocrystalline thin films exhibit enhanced mechanical and physical properties compared to that of its microcrystalline thin films. Particularly, nanocrystalline TiN thin film has been identified as a potential material for tribological and diffusion barrier applications due to its very high hardness, wear resistance, and metallurgical stability, and adhesion properties (Helmerson et al 1987; Patscheider 2003; Barnett and Madan 2004). Patsalas et al (2000) studied the effect of substrate temperature and biasing on the mechanical properties of sputtered TiN thin films by nanoindentation technique and reported that the superior hardness and elastic behaviour are exhibited by the denser films with (200) orientation. The substrate biasing and temperature had a direct influence on obtaining the denser TiN films with (200) orientation. The effect of different substrates such as stainless steel, Ti, brass on the mechanical properties of TiN coatings was studied by nanoindentation technique (Hainsworth and Soh 2003) and it was observed that the indentation response was plastically dominated. The hardness values of the films are scattered at lower load and it was due to inaccuracies manifested in evaluation of load-displacement curves. The elastic recovery and pile-up around the indentations

*Author for correspondence (rjayafmt@iitr.ernet.in) influenced the hardness values of TiN films deposited on the different substrates. The mechanical properties of TiN coatings deposited on different steel substrates were studied using nanoindentation and nanoscratch tests by Zhang and Huan (2004) and it was shown that with the increase in indentation depth, the hardness value decreases due to the substrate effects. The deformation mechanisms of TiN coatings on steel substrates, during nanoindentation, were investigated by focused ion beam imaging and TEM (Ma et al 2005, 2007). It was reported that the loaddisplacement data displayed a pop-in effect with the increase in load on the samples. The cracks were initiated at the boundaries between the columnar grains as well as the steps at the coating/substrate interface. Nanoindentation and microscopic observation revealed that the deformation of TiN coatings occurs primarily by the shear sliding along columnar grain boundaries. Ma et al (2006) investigated the nanohardness of nanocrystalline TiN thin films, prepared by ion-beam assisted deposition, and found that the nanohardness is not sensitive to the packing factor or $\mathrm{N} / \mathrm{Ti}$ ratio but it increases with increasing ion beam energies.

TiN thin films deposited on glass substrate require a detailed investigation to substantiate the influence of microstructural characteristics on deformation mechanisms. It has been reported that TiN films with more than $200 \mathrm{~nm}$ thickness were not mechanically stable on glass substrates and peeled-off up with time on account of stress 
(Massiani et al 1990). It is very essential to prepare mechanically stable thick TiN films on glass substrate and also measure its mechanical properties. In order to ensure compatibility and reliability of TiN thin films in diffusion barriers and tribological applications, the influence of processing conditions on the microstructural characteristics of thin films (Soriaga and Stickney 2002) need to be thoroughly understood. Owing to these facts, the present work has been focused to measure the hardness and modulus of elasticity of d.c. sputtered nanocrystalline TiN thin films by nanoindentation technique. Nanoindentation technique is an effective tool to measure the elastic and plastic properties of the coatings as reported in the literature (Weppelmann and Swain 1996; Shojaei and Karmi 1998). XRD, FE-SEM and AFM techniques were used to characterize the films deposited under different processing conditions. The mechanisms contributing to the improved strength of the films were explained using its microstructural features.

\section{Experimental}

\subsection{Deposition of TiN thin films}

TiN thin films were deposited on glass substrates by d.c. magnetron reactive sputtering. The substrate is a microscope glass slide, which is cleaned by rinsing in ultrasonic baths of acetone and methanol and dried under nitrogen gas. The sputtering target was a $99.99 \%$ pure $\mathrm{Ti}$ disc ( 2 " diameter and $5 \mathrm{~mm}$ thick). The base pressure was better than $2 \times 10^{-6}$ Torr and the sputtering was carried out in an $\mathrm{Ar}+\mathrm{N}_{2}$ atmosphere with the ratio $70: 30$. Before starting the deposition, the target was pre-sputtered for 15 min with a shutter located in between the target and the substrate. All the deposition was performed at a fixed substrate to target distance of $50 \mathrm{~mm}$. The sputtering power of $150 \mathrm{~W}$ is kept fixed during all depositions. The TiN films of different thicknesses $(0 \cdot 31-2 \cdot 83 \mu \mathrm{m})$ were prepared at 10 mTorr working pressure with a deposition temperature of $500^{\circ} \mathrm{C}$.

\subsection{Characterization}

XRD (Bruker AXS, D8 Advance) measurements were made using $\mathrm{CuK} \alpha(\lambda=1.54 \AA)$ radiation to characterize the TiN thin films. The scan rate used was $1 \%$ min and the scan range was from $35^{\circ}-65^{\circ}$. The grain size of the thin films was estimated from the Scherrer's formula, as given in (1). In this expression, the grain size, $t$, is along the surface normal direction, which is also the direction of the XRD diffraction vector.

$$
t=\frac{0 \cdot 9 \lambda}{B \cos \theta}
$$

where $B$ is the corrected full-width at half maximum (FWHM) of a Bragg peak, $\lambda$ the X-ray wavelength, and $\theta$ the Bragg angle. $B$ is obtained from the equation

$$
B^{2}=B_{\mathrm{r}}^{2}-B_{\text {strain }}^{2}-C^{2} \text {, }
$$

where $B_{\mathrm{r}}$ is the FWHM of a measured Bragg peak, $B_{\text {strain }}=\varepsilon \tan \theta$ the lattice broadening from the residual strain, $\varepsilon$, measured by XRD using the $\cos ^{2} \alpha \sin ^{2} \psi$ method, and $C$ the instrumental line broadening (Warren and Biscce 1938). The texture coefficients of the TiN films as a function of deposition time are calculated from their respective XRD peaks using the following formula (Chawla et al 2008)

$$
\text { Texture coefficient }=\frac{I(h k l)}{[I(111)+I(200)+I(220)]},
$$

where $h k l$ represents the (111), (200) or (220) orientations.

Dektak 3030 surface profilometer was used to measure the thickness of TiN films on glass substrate. FE-SEM (FEI, Quanta 200F) were used to characterize the microstructures of the TiN thin films at an acceleration voltage of $20 \mathrm{kV}$ and the surface morphology (2D and 3D) of the TiN films was characterized by AFM (NT-MDT, Ntegra) operated in semi contact (tapping) mode in $3 \times 3 \mu \mathrm{m}^{2}$ scan area to calculate the surface roughness.

\subsection{Nanoindentation measurements}

The hardness and elastic modulus of TiN thin films were measured by using CSM nanoindentation instrument. In this nano hardness tester, an indenter tip with known geometry is driven into a specific site of the material to be tested by applying an increased normal load (Hay and Pharr 2000; Fischer-Cripps 2001). A particular feature of this instrument is the use of a sapphire reference ring that remains in contact with the specimen surface during the indentation. One of the main advantages of this instrument is the ability to quickly and easily make an AFM image of a residual imprint. The precise positioning $(0.5 \mu \mathrm{m})$ capability of the $X-Y$ motorized table combined with the large $X-Y$ ranges $(20 \times 20 \mu \mathrm{m}$ or $40 \times 40 \mu \mathrm{m})$ of the oblective ensures that the indent will always be in the centre of the field of view in both optical and AFM modes. The load is increased and decreased in a linear fashion during the experiment. The diamond Berkovich indenter is forced into thin films being tested under constant load conditions. The loading profile during indentation testing followed linearly increasing with a hold time of $10 \mathrm{~s}$ at the peak load. The loads used were $5 \mathrm{mN}$ at different loading rates $2 \cdot 5,5 \cdot 0,10 \cdot 0,20 \cdot 0$ and $40.0 \mathrm{mN} / \mathrm{min}$ in the present work and the average hardness and Young's modulus values were calculated. Hardness values were consistent $(\delta= \pm 1)$ from indent to indent and uniform over the range of indentation depth of $100-120 \mathrm{~nm}$. The analysis of elastic modulus is based on 

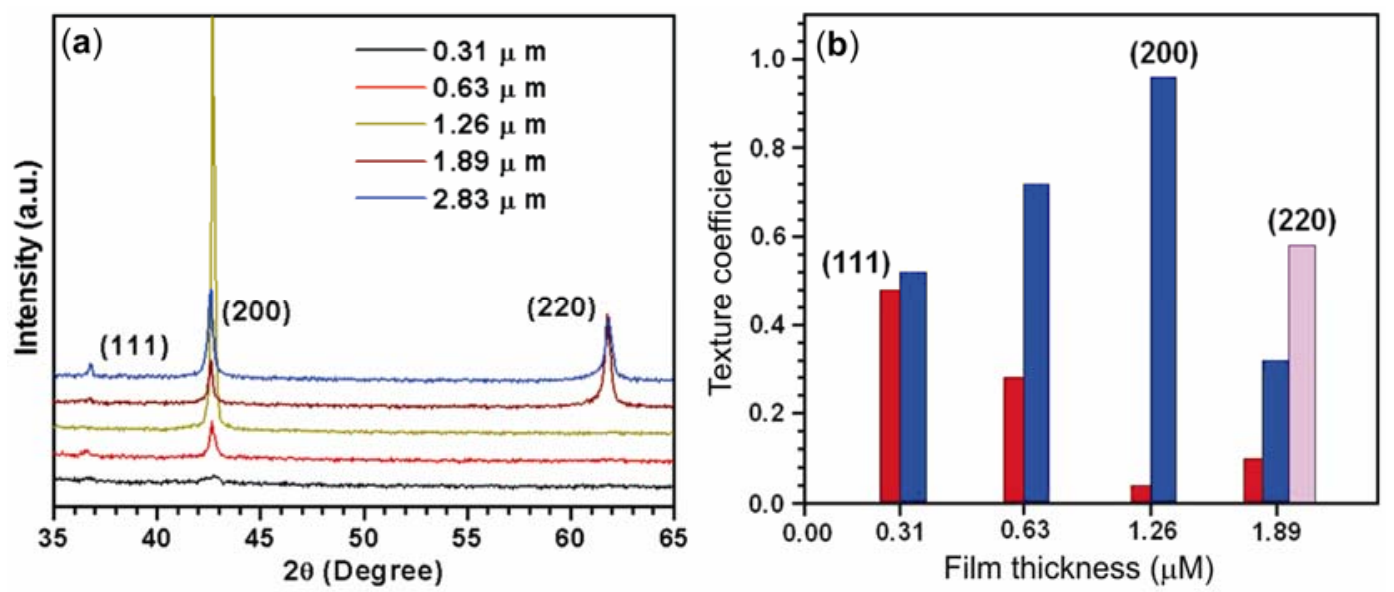

Figure 1. (a) XRD peaks of TiN films deposited on glass substrate and (b) texture coefficients of TiN films deposited on glass substrate.

Table 1. Different properties of TiN films with varying film thickness.

\begin{tabular}{lcccc}
\hline S1. no. & $\begin{array}{c}\text { Film thickness } \\
(\mu \mathrm{m})\end{array}$ & $\begin{array}{c}\text { Crystallite size, } \\
\text { XRD }(\mathrm{nm})\end{array}$ & $\begin{array}{c}\text { Average roughness, } \\
\text { AFM }(\mathrm{nm})\end{array}$ & Micro strain \\
\hline 1. & 0.31 & 8.46 & 7.59 & -0.116 \\
2. & 0.63 & 27.03 & 9.57 & 0.541 \\
3. & 1.26 & 45.12 & 12.43 & 0.002 \\
4. & 1.89 & 29.38 & 11.33 & 0.060 \\
5. & 2.83 & 47.39 & 18.97 & 0.609 \\
\hline
\end{tabular}

the Oliver and Pharr method (Oliver and Pharr 1992). The modulus of a material was calculated from the loaddisplacement plots by modeling the unloading curve with a power law relationship instead of assuming linear unloading. At any time during loading, the total displacement, $h$, is written as

$$
h=h_{\mathrm{c}}+h_{\mathrm{s}},
$$

where $h_{\mathrm{c}}$ is the vertical distance through which contact is made (contact depth) and $h_{\mathrm{s}}$ the displacement of the surface at the perimeter of the contact. The quantity, $h_{\mathrm{s}}$, depends on the indenter geometry. From Sneddon's solution for a conical indenter, $h_{\mathrm{s}}$ becomes

$$
h_{\mathrm{s}}=\frac{(\pi-2)}{\pi}\left(h-h_{\mathrm{f}}\right)
$$

where $h_{\mathrm{f}}$ is the final depth upon unloading. The quantity $h-h_{\mathrm{f}}$ appears rather than $h$ by itself since Sneddon's solution applies only to the elastic component of the displacement. The contact depth, $h_{\mathrm{c}}$, is expressed as

$$
h_{\mathrm{c}}=h_{\mathrm{t}}-\stackrel{\circ}{a} \frac{P_{\max }}{\mathrm{d} P / \mathrm{d} h}
$$

where $\mathrm{d} P / \mathrm{d} h$ is stiffness, i.e derivative of a power law fit to the unloading curve and evaluated at the maximum load, $P_{\max }, h_{\mathrm{t}}$ the maximum depth, and $\stackrel{a}{\text { refers to tip }}$ shape constant and is equal to 0.72 for a conical tip and 1 for a flat punch. The area function for a perfect Berkovich tip is given as

$$
A=24 \cdot 5 h_{\mathrm{c}}^{2} \text {. }
$$

The hardness is calculated using the expression

$$
H=\frac{P_{\max }}{A_{\mathrm{c}} f\left(h_{\mathrm{c}}\right)} .
$$

The reduced elastic modulus $\left(E_{\mathrm{r}}\right)$ is calculated from

$$
E_{\mathrm{r}}=\frac{\mathrm{d} P}{\mathrm{~d} h} \frac{1}{2} \frac{\sqrt{\pi}}{\sqrt{A}} .
$$

\section{Results and discussion}

The XRD peaks for the TiN films deposited, at $500^{\circ} \mathrm{C}$, are shown in figure 1(a). It is observed that the preferred orientation of the film, with increasing thickness, is (200) up to $1.26 \mu \mathrm{m}$ due to the lowest surface free energy during film growth in comparison to strain energy (Chawla et al 2008 ), but as the film thickness increases above $1.23 \mu \mathrm{m}$ competition between surface energy and strain energy increases and hence (220) peak develops to minimize the total energy (Hoang et al 1996). With an increase in 
(a)

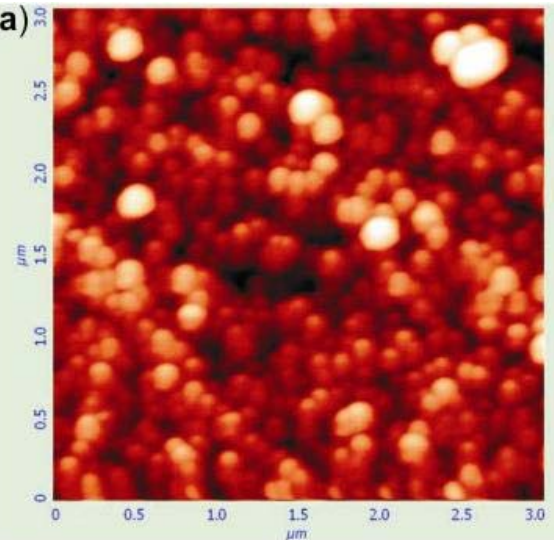

(c)

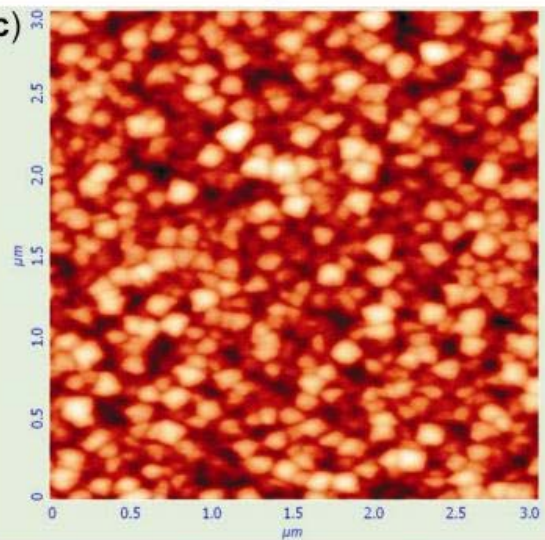

$(\mathbf{e})$

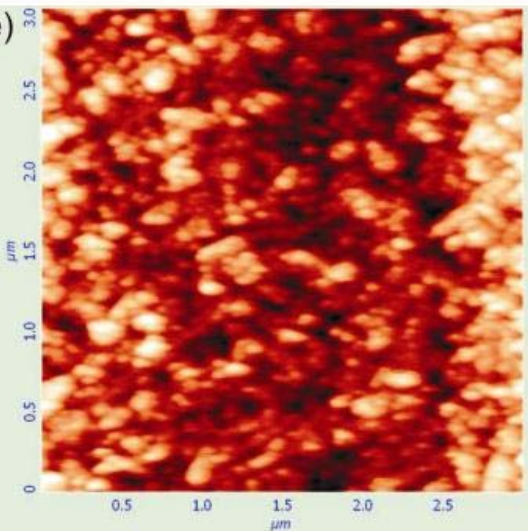

(b)
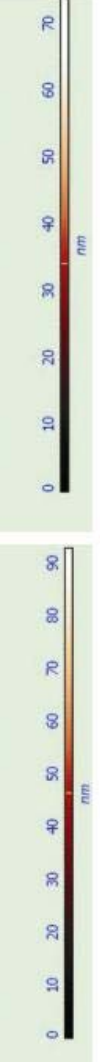

(d)
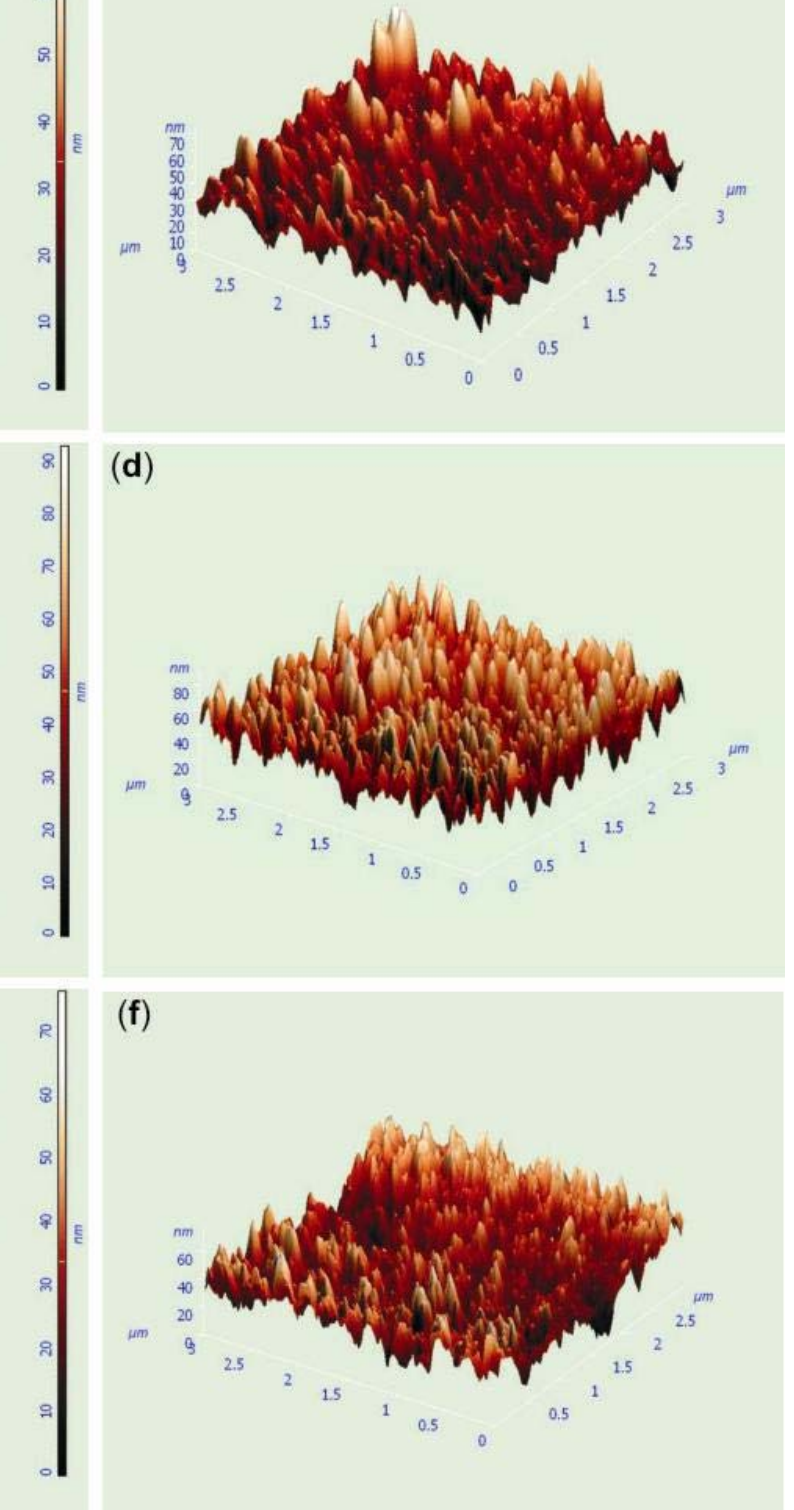

(f)

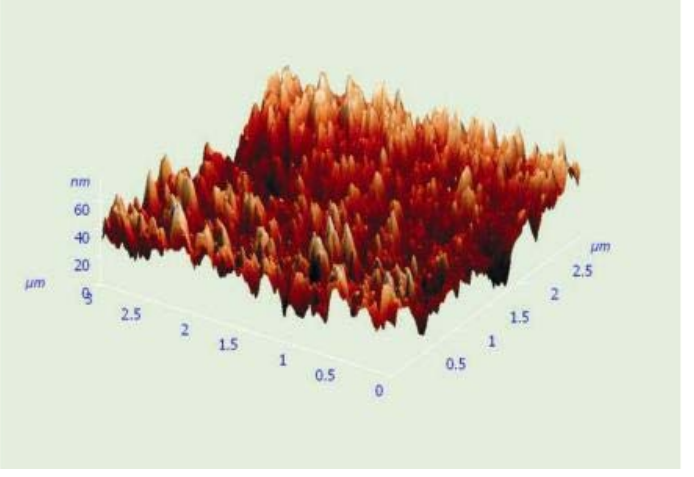

Figure 2. 2D and 3D AFM images of TiN films deposited on glass substrate at thicknesses (a) \& (b) $0.63 \mu \mathrm{m}$, (c) \& (d) $1.26 \mu \mathrm{m}$ and (e) \& (f) $1.89 \mu \mathrm{m}$.

deposition time and at some critical thickness, stress relaxation may reduce strain energy (Venables 2000; Ohring 2002) to a greater extent than the increase in surface energy due to the texture changes. It is well known that the anisotropy of the elastic moduli will favour the growth of low strain energy-oriented grains at the expense of grains possessing higher strain energy (Chawla et al 2008). Table 1 shows the variation of crystallite size of TiN films as a function of its thickness values. The crystallite size of the thin film with $0.31 \mu \mathrm{m}$ thickness, calculated from the X-ray peak broadening, is $8.46 \mathrm{~nm}$. It increases with increase in thickness but for the films with
$1.89 \mu \mathrm{m}$ thickness, the crystallite size is smaller as shown in table 1. Figure 1(b) shows the texture coefficients of the TiN films as a function of film thickness calculated from their respective XRD peaks by using (2). It is observed that the texture coefficient of (200) peak increases till the film reaches a thickness of $1.26 \mu \mathrm{m}$ and afterwards (220) orientation develops.

Figure 2 shows the 2D and 3D AFM images of the TiN films of $0.63 \mu \mathrm{m}, 1.26 \mu \mathrm{m}$ and $1.89 \mu \mathrm{m}$ thicknesses. The surface roughness of the films increases with increasing thickness but for the film with thickness of $1.89 \mu \mathrm{m}$, a low surface roughness value is observed as shown in table 1. 

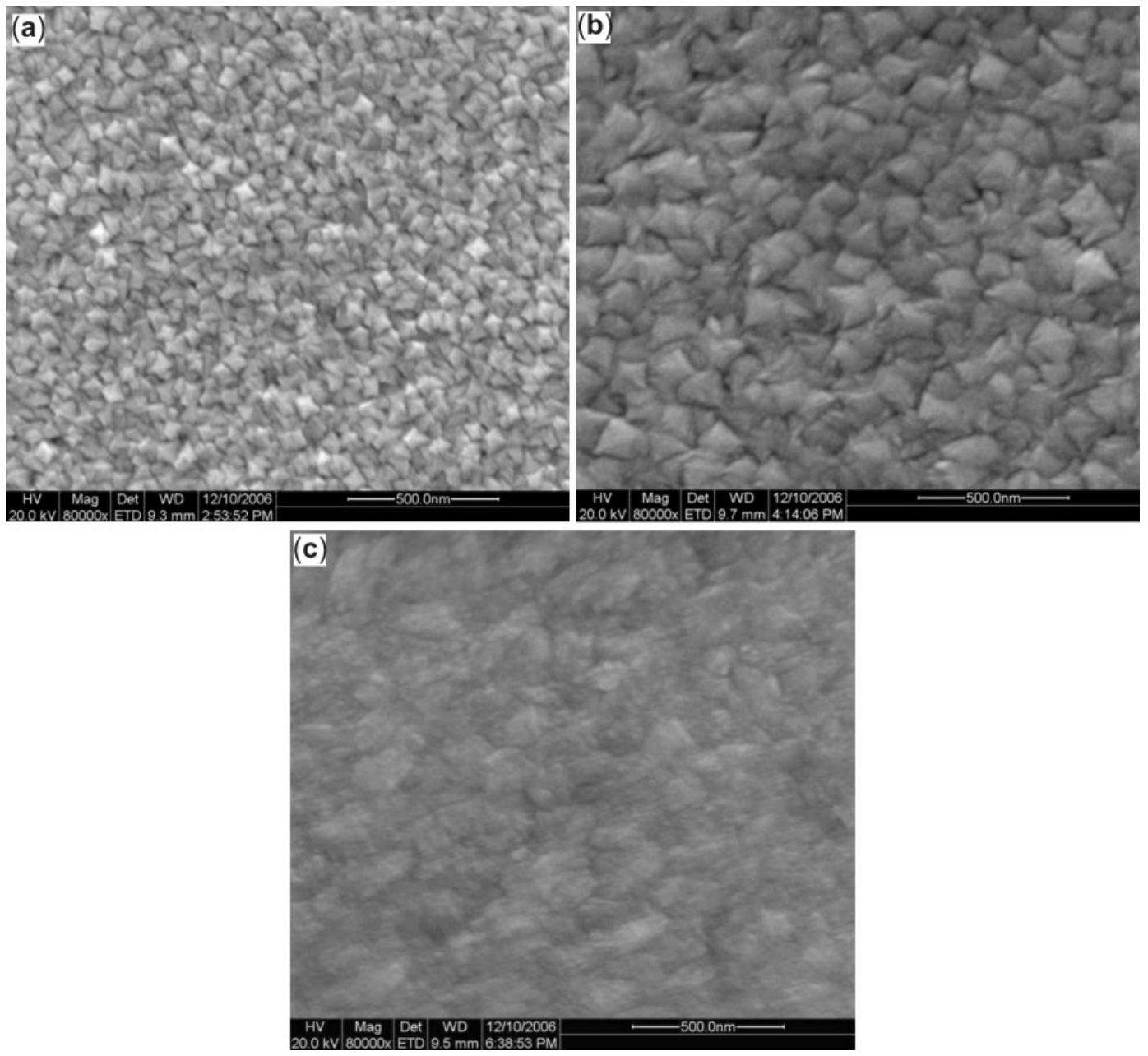

Figure 3. FESEM images of TiN films deposited on glass substrate at thicknesses (a) $0.63 \mu \mathrm{m}$, (b) $1.26 \mu \mathrm{m}$ and (c) $1.89 \mu \mathrm{m}$.

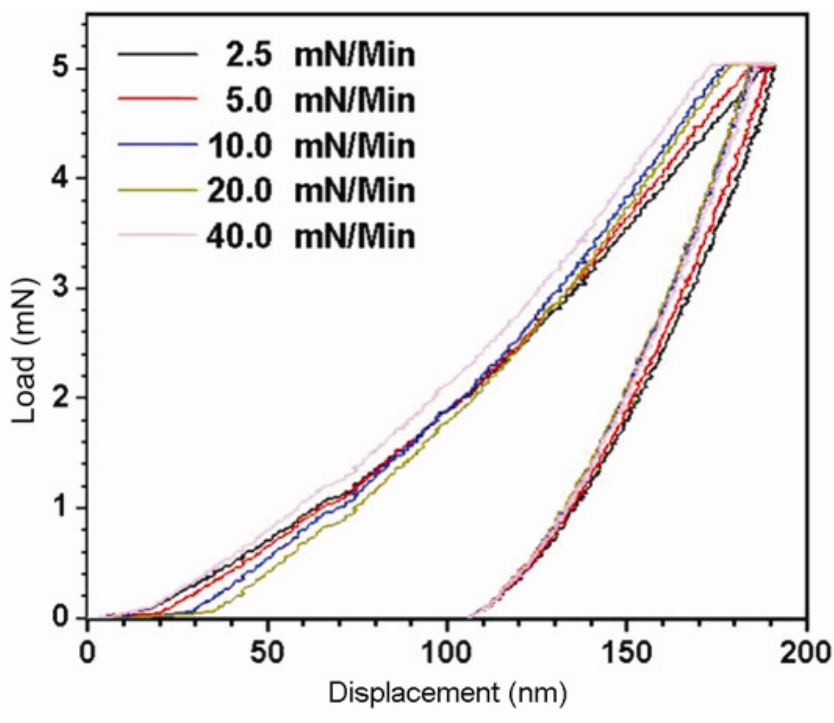

Figure 4. Load-displacement curve of TiN thin films deposited on glass substrate.
The FE-SEM image of the TiN films of $0.63 \mu \mathrm{m}$, $1.26 \mu \mathrm{m}$ and $1.89 \mu \mathrm{m}$ thicknesses are shown in figure 3 . The preferred orientation of grains with $(200)$ texture in TiN thin films is evident from the XRD peaks up to thickness $1.26 \mu \mathrm{m}$ and it exhibits columnar grain shape as observed from the FE-SEM images in figures 3(a) and (b). However, thin films with $1.89 \mu \mathrm{m}$ exhibit diffused grain structure when (220) peak dominates as shown in figure 3(c). The load-displacement curve obtained during nanoindentation testing of the TiN thin films in different loading rates $(2 \cdot 5,5 \cdot 0,10 \cdot 0,20 \cdot 0$ and $40.0 \mathrm{mN} / \mathrm{min})$ is shown in figure 4 . The maximum load used was $5 \mathrm{mN}$ at different loading rates used in the present work. For each sample, 15 measurements were performed at different loading rates and the average value was reported.

Figure 5(a) shows the influence of thickness on hardness of TiN thin films. The hardness value increases with increasing film thickness from $0.3 \mu \mathrm{m}$ to $1.89 \mu \mathrm{m}$ but it drops for $>2.5 \mu \mathrm{m}$ thick films. The texture coefficient of TiN thin films increases with thickness due to the pre- 
ferred orientation (220) of the films, which in turn reflects in higher hardness value. The influence of preferred orientation (220) of the grains on the higher hardness value can be explained by relating it with the resolved shear stress on the TiN slip system. The higher hardness of TiN films with (220) with preferred orientations may be attributed to very fine grain size $(18.46 \mathrm{~nm})$ with its less active slip system. The drop in hardness value of TiN thin film with thickness $>2.5 \mu \mathrm{m}$ may be due to higher surface roughness of the film.

The Young's modulus $(E)$ of TiN thin films is shown in figure 5(b). It is evident that $E$ increases with thickness due to increased anisotropy of the film with (220) preferred orientation. The films with higher thickness may favour the textured film as well as the higher packing factor, which could influence the $E$ value significantly. The modulus of elasticity of TiN films is less than that of the standard values cited in the literature due to the porosity of the sputtered films. It is due to the following reasons: (i) pores reduce the cross sectional area of the samples across which a load is applied and (ii) porosity acts as a stress raiser, which decreases the resistance to
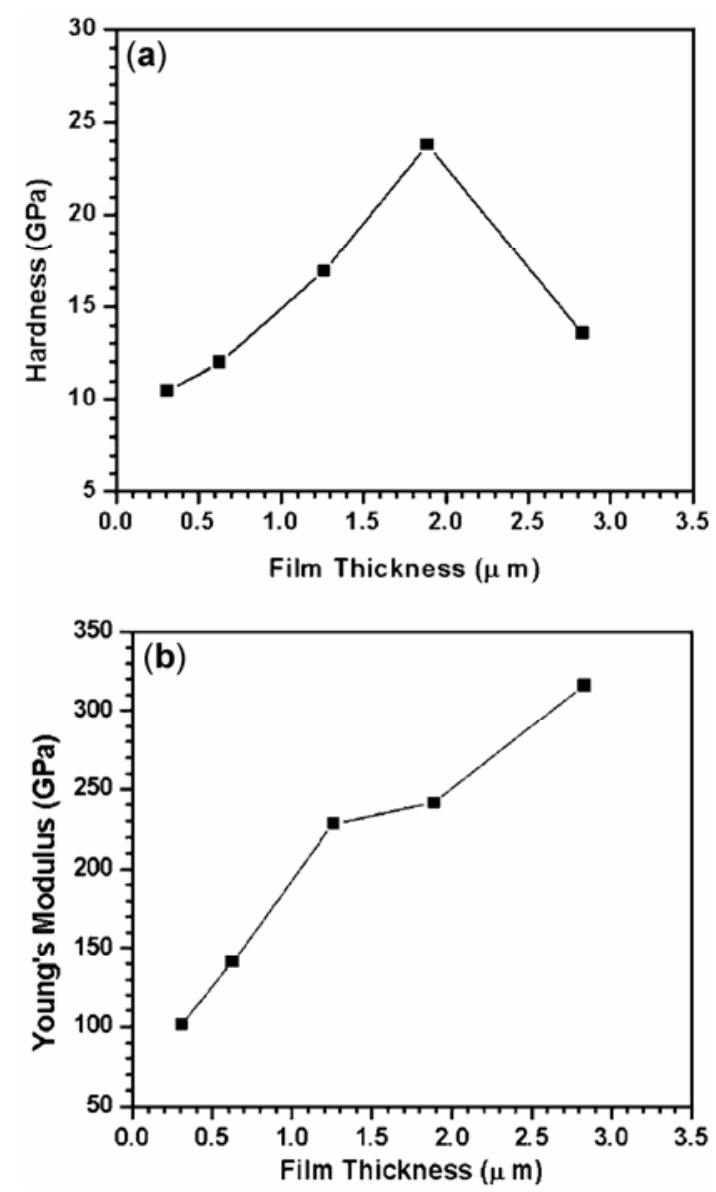

Figure 5. (a) Hardness of TiN thin film as a function of film thickness on glass substrate and (b) Young's modulus of TiN thin film as a function of film thickness on glass substrate. elastic deformation significantly. With increase in film thickness, the porosity of the films would increase depending upon the growth of columnar grains with voids, influenced by the deposition conditions such as working pressure, deposition temperature, and bias voltages. The porosity $(P)$ of the films can be calculated by the following equation (Jin et al 1995)

$$
P=\left(d_{\mathrm{a}}-d_{\mathrm{m}}\right) / d_{\mathrm{a}},
$$

where $d_{\mathrm{a}}$ is the apparent thickness of the films and $d_{\mathrm{m}}$ the mass thickness, i.e. theoretical thickness of the films without porosity. With increase in apparent thickness, the porosity of the film would increase as evident from the equation.

Table 1 shows the average micro strain obtained from (111), (200) and (220) peaks of TiN film on glass substrate which were calculated by the following equation (Ong et al 2002; Singh and Kaur 2008)

$$
\varepsilon=\left(c-c_{0}\right) / c_{0} \times 100,
$$

where $c$ is the lattice parameter of the strained TiN films calculated from XRD data and $c_{0}$ the unstrained lattice parameter of $\operatorname{TiN}(4.23 \AA)$. The lattice parameter, $c$, was calculated by equaling the interplanar spacing, $d$, obtained from the position of the (111), (200) and (220) peaks using the Bragg condition. It was found that initially micro strain was negative and after that all values were positive with increasing thickness. The highest hardness was found for the TiN films with thickness $1.26 \mu \mathrm{m}$ and $1.89 \mu \mathrm{m}$ and it showed less positive micro strain.

\section{Conclusions}

Nanocrystalline TiN thin films were deposited on glass substrate by d.c. magnetron sputtering. The effect of film thickness on hardness and $E$ was investigated by nanoindentation technique. The formation of nanosized crystallites in thin films has been confirmed from the XRD, AFM and FE-SEM characterizations. The preferred orientation of TiN film is (200) up to thickness $1.26 \mu \mathrm{m}$ and (220) and (200) peaks dominate, respectively with increase in thickness up to $2.83 \mu \mathrm{m}$. It is because of the competition between surface energy and strain energy during film growth. The hardness of TiN thin film increases with increase in thickness up to $1.89 \mu \mathrm{m}$ (maximum hardness of $24 \mathrm{GPa}$ ) but it drops for $>2.5 \mu \mathrm{m}$ thick films. There was no drop in modulus of elasticity and it increases with increase in film thickness. The TiN films with highest hardness exhibits a less positive micro strain.

\section{Acknowledgements}

Two of the authors (RC and VC) would like to thank DST and DRDO, for financial support. 


\section{References}

Barnett S A and Madan A 2004 Scr. Mater. 50739

Chawla V, Jayaganthan R and Chandra R 2008 Mater. Charact. 591015

Fischer-Cripps 2001 Nanoindentation, mechanical engineering series (New York: Springer)

Hainsworth S V and Soh W C 2003 Surf. \& Coat. Technol. 163-164515

Hay J L and Pharr G M 2000 Mater. Test. \& Eval. 8232

Helmerson U, Todorova S, Barnett S A, Sundgren J E, Market L C and Greene J E 1987 J. Appl. Phys. 62481

Hoang N H, McKenzie D R, McFall W D and Yin Y 1996 J. Appl. Phys. 806279

Jin P, Nako S, Tanemura S and Maruno S 1995 Thin Solid Films 27119

Ma C H, Huang J H and Chen H 2006 Surf. \& Coat. Technol. 2003868

Ma L W, Cairney J M, Hoffman M and Munroe P R 2005 Surf. \& Coat. Technol. 19211
Ma L W, Cairney J M, Hoffman M and Munroe P R 2007 Thin Solid Films $\mathbf{5 1 5} 3190$

Massiani Y, Medjahed A, Gravier P, Argeme L and Fedrizzi L 1990 Thin Solid Films 191305

Ohring M 2002 Materials science of thin films (San Diego: Academic Press)

Oliver W C and Pharr G M 1992 J. Mater. Res. 71564

Ong H C, Zhu A X E and Du G T 2002 Appl. Phys. Lett. 80941

Patsalas P, Charitidis C and Logothetidis S 2000 Surf. \& Coat. Technol. 125335

Patscheider J 2003 MRS Bull. 28180

Shojaei O R and Karmi A 1998 Thin Solid Films 332208

Singh P and Kaur D 2008 J. Appl. Phys. 1031

Soriaga M P and Stickney J 2002 Thin films: preparation, characterization, applications (New York: Kluwer Academic)

Venables John A 2000 Introduction to surface and thin film processes (Cambridge: Cambridge University Press)

Warren B E and Biscce 1938 J. Am. Ceram. Soc. 2149

Weppelmann E R and Swain M V 1996 Thin Solid Films 286111

Zhang T H and Huan Y 2004 Tribol. Lett. 17911 